\title{
Wavelength effects on simple reaction time
}

\author{
MARY JO NISSEN and JOEL POKORNY \\ Eve Research Laboratories, The University of Chicago, Chicago, Illinois 60637
}

\begin{abstract}
Simple reaction time was measured to spectral lights matched photometrically in luminance. When these lights were presented on a dimmer achromatic background, reaction time did not vary as a function of wavelength. Moreover, reaction times to white and chromatic lights were the same. When the luminance of the background was the same as that of the chromatic lights, reaction time increased and showed a strong effect of wavelength. Reaction time in this condition appeared to follow a saturation function. The results are described in terms of the operation of achromatic and chromatic processing channels.
\end{abstract}

Previous data on the effect of wavelength on simple reaction time (RT) have been in remarkable accord: when chromatic lights are matched in luminance at photopic levels, and when these lights are presented on a dimmer, achromatic background, simple RT does not vary with wavelength (Finn \& Lit, 1971; Holmes, 1926; Lit, Young, \& Shaffer, 1971; Mollon \& Krauskopf, 1973; Pollack, 1968).

Because the chromatic stimuli in these experiments appeared on a less luminant background, there were actually two cues accompanying stimulus onset on which subjects could base a response. They could respond to the luminance increment or to the chromatic signal. If they used the former cue, one might not expect a wavelength effect, inasmuch as the size of this luminance increment was matched for all wavelengths.

One way to ensure that subjects respond on the basis of the chromatic signal is to use a disjunctive RT task. Jones and Wilkinson (1975) presented subjects with equally luminant white, red, or green stimuli on a dark background. Subjects were instructed to respond only to the chromatic lights and to withhold responses to the white light. They were thus unable to respond on the basis of the luminance signal which was present on every trial. The results showed that RT to green $(510 \mathrm{~nm})$ was about $24 \mathrm{msec}$ slower than that to red $(645 \mathrm{~nm})$.

An alternative way to isolate the effect of color on RT is to match the luminance of the target stimuli and the background so that no luminance change accompanies stimulus onset. When Pieron (1931) measured simple RT under these conditions, he found that RT was shorter to red than to green or blue. However, neither the details of his luminance

This work was supported in part by NIH, USPH, NEI Girants EY 70652, EY 00901, EY 07010 (J. Pokorny) and EY 00523 (F. W. Newell). Requests for reprints should be sent to Mary Jo Nissen. Eye Research Laboratories. The University of Chicago. 950 East 59th Street, Chicalen, Illinois 60637. matching procedure nor the level of luminance was reported.

Some recent studies, using other techniques to measure the effect of wavelength on visual latency, have compared conditions in which the chromatic stimulus is accompanied by a luminance change and those in which it is not. Breton (1977) and Weingarten (1972) found that when subjects made temporal order judgments to chromatic lights presented on a dimmer achromatic background, there was no effect of wavelength. However, when the chromatic stimuli and the background were matched in luminance, the relative latency for red was less than that for green. In Weingarten's data, the difference was 20 to $25 \mathrm{msec}$. Bowen, Lindsey, and Smith (Note 1), measuring two-pulse resolution thresholds, found no wavelength effect for chromatic stimuli accompanied by a luminance cue; when the stimuli and background were of equal luminance, temporal resolution was poorest for yellow $(570 \mathrm{~nm})$ and best for blue $(463 \mathrm{~nm})$ and red $(620 \mathrm{~nm})$.

The present experiments measure simple RT to chromatic stimuli presented with and without luminance cues. The term "substitution" will refer to the condition in which the stimuli and background are matched in luminance: a chromatic stimulus is "substituted" for an equally luminant achromatic background. In the increment condition, the luminance of the background is reduced by $0.3 \log$ unit, and the onset of the chromatic stimulus is thus accompanied by a luminance change. All chromatic stimuli were photometrically matched in luminance and were of the same luminance in the substitution and increment conditions.

\section{EXPERIMENT 1}

Simple RT to seven wavelengths was determined in substitution and increment conditions. RT to a white light was also measured in the increment condition. 


\section{Method}

Subjects. Two men (K.H. and Y.C.) and one woman (M.J.N.), each in the 20 s, served as subjects. Each had normal color vision, as determined by the Nagel anomaloscope and FM 100-Hue test, and normal visual acuity with corrective lenses. M.J.N. (the first author) was experienced in RT tasks, whereas K.H. and Y.C. were naive subjects and had no previous experience in RT tasks. K.H. and Y.C. were each paid $\$ 2.50 / \mathrm{h}$ for their participation.

Apparatus. The stimulus display consisted of a $3.8^{\circ}$ circular achromatic background field. Target stimuli appeared as a circular field of $1.9^{\circ}$ centered on the background field. The array was viewed monocularly in darkness at a distance of $0.4 \mathrm{~m}$ with the subject's head positioned by a chinrest. The subjects were instructed to fixate the center of the display.

The display was produced by a three-channel optical projection system (Figure 1). A 150-W xenon arc lamp was the source (S) for all three channels.

Channel A produced the chromatic and achromatic target stimuli. Light passed through neutral density filters, and for the chromatic stimuli through interference filters, which were placed in either filter box 1 (FB1) or filter box 2 (FB2). The light was focused at shutter 1 (SH1) and illuminated diffusing screen 1 (DS1). Channels B and C together produced the achromatic background field. Light in channel $\mathrm{B}$ was focused at shutter 2 (SH2) and illuminated DS1. Light in channel $C$ was collimated and illuminated diffusing screen 2 (DS2). Channel B produced the center portion of the background corresponding to the target stimulus position. Channel $C$ produced the remaining annular portion of the background field. A piece of nonglare glass $(\mathrm{G})$ between DS2 and the subject $(O)$ served to produce a slight blur of the border which would otherwise appear between the center and surround of the achromatic background. This blur succeeded in making the background field appear completely homogeneous.

Target stimuli were presented by, first, activating a motor which moved either FB1 or FB2 into the beam of channel A. Then $\mathrm{SH} 2$ was closed and $\mathrm{SH} 1$ was opened simultaneously. The chromatic beam of channel A thus replaced the center portion of the achromatic background. The shutters consisted of opaque flags mounted on loudspeaker coils which were driven by $\pm 10-\mathrm{V}$ pulses. The rise and fall times of these shutters were less than $4 \mathrm{msec}$. Although the shutters operated very quietly, subjects wore sound-deadening earmuffs to ensure that no auditory cues accompanied stimulus onset.

This optical apparatus was interfaced to a PDP-15 computer which was programmed to control the positions of FB1 and $\mathrm{FB} 2$, the opening and closing of $\mathrm{SH} 1$ and $\mathrm{SH}$, and all timing. It also measured and recorded reaction times. In a word, the experiment was computer-controlled.

A calibrated laboratory-constructed spectroradiometer was used to determine the spectral character of the achromatic background and of the interference filters used to produce the chromatic lights. The achromatic background had chromattcity coordinates of $x=.3607, y=.3616$, and a correlated color temperature of $4505^{\circ} \mathrm{K}$. Its luminance, measured with an Ilford photometer, was $0.7 \mathrm{~cd} / \mathrm{m}^{2}$, producing an estimated retinal illuminance of 9.3 trolands (drGroot \& Gebhard, 1952). The peak wavelength and half-transmission bandwidth of the narrow-band interference filters, measured in the apparatus, follow: $430 \mathrm{~nm}$ (halftransmission bandwidth, $8 \mathrm{~nm}), 460 \mathrm{~nm}(7 \mathrm{~nm}), 502 \mathrm{~nm}(17 \mathrm{~nm})$, $542 \mathrm{~nm}(9 \mathrm{~nm}), 570 \mathrm{~nm}(11 \mathrm{~nm}), 613 \mathrm{~nm}(11 \mathrm{~nm})$, and $650 \mathrm{~nm}$ $(10 \mathrm{~nm})$. Although the interference filters were in a converging beam, the maximal deviation from orthogonal light entry was only $4^{\circ}$. The measured peak wavelengths and half-transmission bandwidths differed only trivially from Cary 14 spectrophotometric measurements.

Procedure. Prior to the collection of RT data, the luminance of each chromatic stimulus was matched to that of the background by heterochromatic flicker photometry. The center portion of the background (channel B) and the chromatic light (channel A) were temporally alternated by activating the shutters at fre-

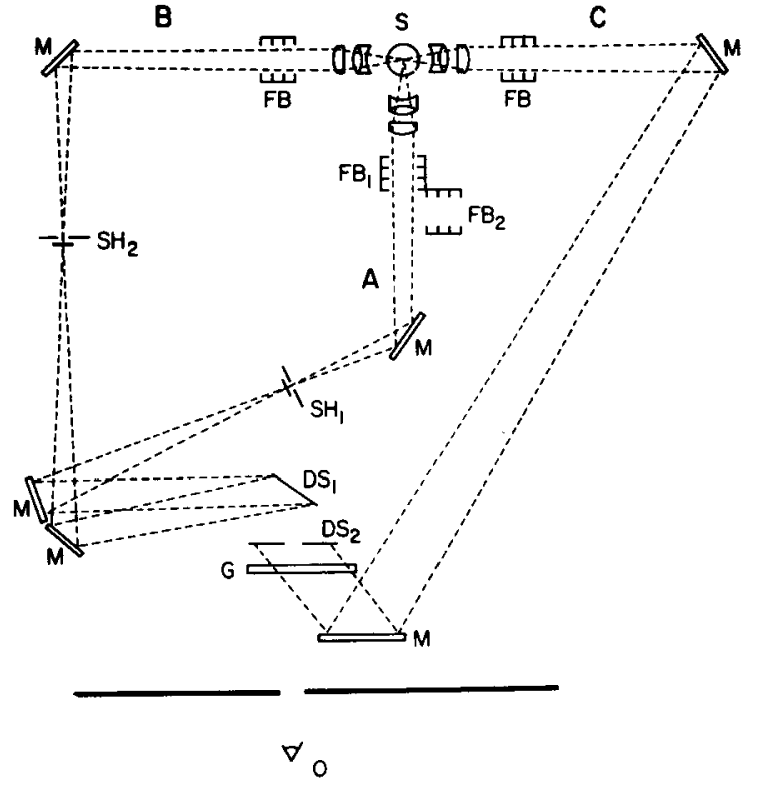

Figure 1. Three-channel (A, B, C) optical system: S, source; FB, filter box; $M$, mirror; SH, shutter; DS, diffusing screen; $G$, nonglare glass; $O$, subject.

quencies ranging from 6 to $10 \mathrm{~Hz}$. Neutral density was added to the chromatic beam until minimal flicker was present.

This flicker procedure ensured that the luminance of the background field and all chromatic stimuli in the substitution condition of the RT task were matched for each subject. The absence of an artifactual luminance transient upon stimulus presentation was verified by slowly alternating between the background and an equally luminant achromatic target stimulus. With a $570-\mathrm{nm}$ interference filter placed before the entire field, no alternation was detected. In the increment condition, the luminance of the center portion of the background was reduced by $0.3 \log$ unit by adding neutral density in channel $\mathbf{B}$. However, the luminance of the annular portion of the background (channel $C$ ) and that of all chromatic stimuli remained the same as in the substitution condition.

Trials in the RT task were grouped into 40-trial blocks, each of which included 20 presentations of each of two target stimuli. The interference and appropriate neutral density filters for one stimulus were placed in FB1; those for the other stimulus were placed in FB2. Subjects initiated a block of trials with a single keypress.

At the beginning of each trial, the motor controlling the positions of FB1 and FB2 was in a neutral position. After one of the two target stimuli was randomly selected for presentation, the motor positioned either FB1 or FB2 into the beam of channel $A$. The voltage delivered to the motor varied randomly between trials so that the sound of the motor did not indicate which stimulus had been selected. Following the movement of the filter boxes, which took approximately $3 \mathrm{sec}$, was a blank foreperiod of $500,1,000,1,500,2,000 \mathrm{msec}$. Since subjects were able to detect the sound of the filter box motor, the termination of this sound served as a warning cue marking the beginning of the foreperiod. Stimulus onset occurred at the end of the foreperiod. Subjects were instructed to respond as quickly as possible to either stimulus by pressing a telegraph key with their preferred hand. Stimuli were response-terminated. At the end of the trial, the filter box motor returned to its neutral position. No feedback was provided.

In the substitution condition, two of the seven chromatic stimuli were presented in each block. Every possible pair was used once, 
making a total of 21 blocks. Each subject thus responded to each wavelength 120 times in the substitution condition. In the increment condition, eight target stimuli were used (seven chromatic lights and a white light of equal luminance), making a total of 28 blocks with 140 responses per stimulus per subject. The order of blocks in each condition was random, with the restriction that each stimulus was tested at least once on every day of the experiment.

The subjects performed six practice blocks on Day 1. Six blocks lasting $1 \mathrm{~h}$ were performed on each of the following days of the experiment. The subjects ran in substitution and increment condilions on alternate days.

\section{Results}

Mean reaction times were computed for each subject. Individual mean RT data are presented in Figure 2. Error bars are drawn to indicate $95 \%$ confidence limits. Median RT data were also computed but did not differ substantially from the means. The rate of anticipatory responses (those occurring before stimulus onset) was $2 \%$ for M.J.N. and Y.C., and $1 \%$ for K.H. All trials on which there was an anticipation were excluded from analysis.

There are three major features of these data: RT is faster in the increment condition than in the substitution condition; the effect of wavelength is minimal in the increment condition but very strong in the substitution condition; in the substitution condition RT is slowest to stimuli of $570 \mathrm{~nm}$, decreases monotonically with wavelengths either longer or shorter

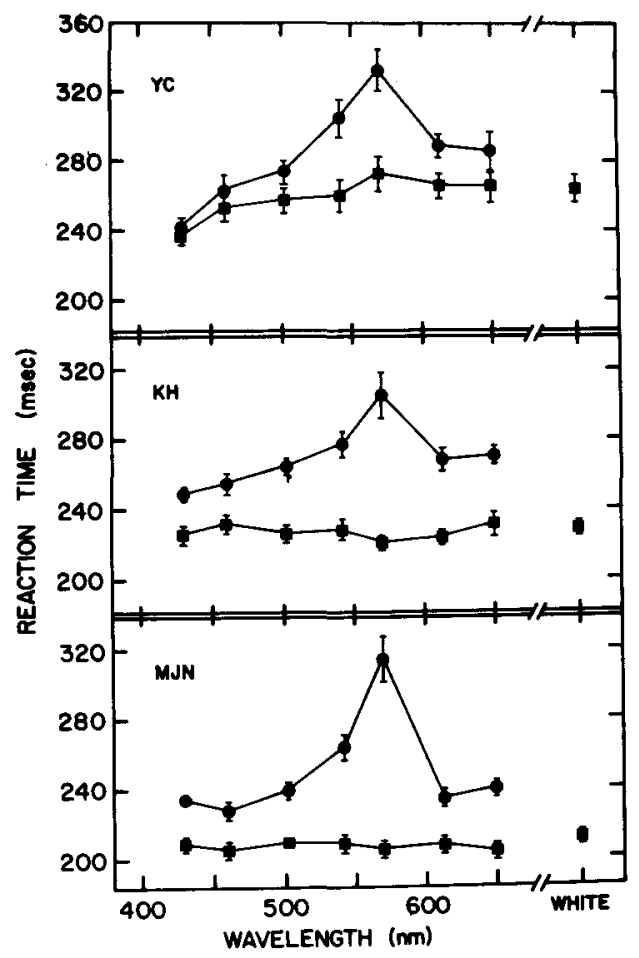

Figure 2. Individual mean reaction time as a function of wavelength in Experiment 1 for substitution (circles) and increment (squares) conditions. than $570 \mathrm{~nm}$, and is somewhat faster to short wavelengths than to long wavelengths.

Mean RT data were subjected to a four-way analysis of variance (ANOVA). Factors included Foreperiod (4 levels) by Wavelength (7) by Condition (2) by Blocks (6). Only responses to the seven chromatic stimuli were included in this ANOVA. Each stimulus occurred in six blocks in the substitution condition, but in seven blocks in the increment condition. For the purpose of this analysis, Blocks 6 and 7 of the increment condition were averaged.

The ANOVA indicated significant main effects of condition $[F(1,2)=38.60, p<.025]$, wavelength $[F(6,12)=7.90, p<.002]$, and foreperiod $[F(3,6)$ $=11.06, \mathrm{p}<.008]$. Reaction time decreased monotonically with foreperiod, from $264 \mathrm{msec}$ following a foreperiod of $500 \mathrm{msec}$ to $237 \mathrm{msec}$ following a $2,000-\mathrm{msec}$ foreperiod. There was a significant interaction between wavelength and condition $[F(6,12)$ $=23.21, \mathrm{p}<.001]$ and between wavelength and foreperiod $[F(18,36)=2.24, p<.02]$. The effect of wavelength was reduced at longer foreperiods. This interaction may have resulted from less temporal uncertainty (leading to more temporally triggered responses) or the use of lower response criteria at long foreperiods.

The interaction between wavelength and condition reflects the much larger effect of wavelength in the substitution condition than in the increment condition. To determine whether there was any significant wavelength effect in the increment condition, a three-way ANOVA was performed on those data. Factors included Foreperiod (4) by Wavelength (8) by Blocks (7). All seven blocks were now included, as were responses to the white light. The ANOVA indicated only a significant main effect of foreperiod $[F(3,6)=8.66, p<.014]$. The effect of wavelength was not significant $[F(7,14)=.652, p>.5]$.

\section{EXPERIMENT 2}

The results of Experiment 1 show that when chromatic stimuli are unaccompanied by a luminance signal, there are systematic effects of wavelength on simple RT. Perhaps most striking is the sharp increase in response latency to light of $570 \mathrm{~nm}$. Experiment 2 investigates responses to this region of the spectrum in more detail. Two new subjects were used to increase the generality of our previous data showing a peak at $570 \mathrm{~nm}$. The stimuli were 560,570 , and $580 \mathrm{~nm}$ lights presented in the substitution condition.

\section{Method}

Subjects. Two men (D.L. and R.W.), who had not participated in Experiment 1, and one woman (M.J.N.), who had, served as subjects. D.L. and R.W., each in the 20 s, had normal color vision and normal visual acuity with corrective lenses. Neither D.L. nor R.W. was experienced in RT tasks. 
Apparatus. The stimulus display and apparatus were the same as in Experiment 1. Interference filters included: $560 \mathrm{~nm}$ (half-bandwidth transmission, $10 \mathrm{~nm}), 570 \mathrm{~nm}(11 \mathrm{~nm})$, and $580 \mathrm{~nm}(9 \mathrm{~nm})$. The luminance of the achromatic background was the same as in Experiment 1.

Procedure. The luminance of each chromatic stimulus was matched for each subject to that of the background by heterochromatic flicker photometry.

The procedure on each trial of the RT task was the same as in Experiment 1. Following one practice session, subjects ran for 5 days. On the first 4 days, each of the three possible pairs of stimuli was tested twice, and on the final day each pair was used once. The order of blocks across days was controlled by a Latin square. There were 360 responses per wavelength per subject.

\section{Results}

Individual mean RT data are shown in Figure 3. The rate of anticipatory responses was $1 \%$ for D.L. and M.J.N., and 3\% for R.W. All subjects responded most slowly to the 570-nm stimulus. A three-way ANOVA (Foreperiod by Wavelength by Blocks) indicated significant main effects of foreperiod $[\mathrm{F}(3,6)=12.53, \mathrm{p}<.006]$ and wavelength $[F(2,4)=13.10, p<.018]$. No interactions were significant.

These data confirm that when chromatic stimuli are presented without a luminance cue, RT is slowest to wavelengths near $570 \mathrm{~nm}$. The results also suggest that RT is quite sensitive to wavelength differences in this region of the spectrum.

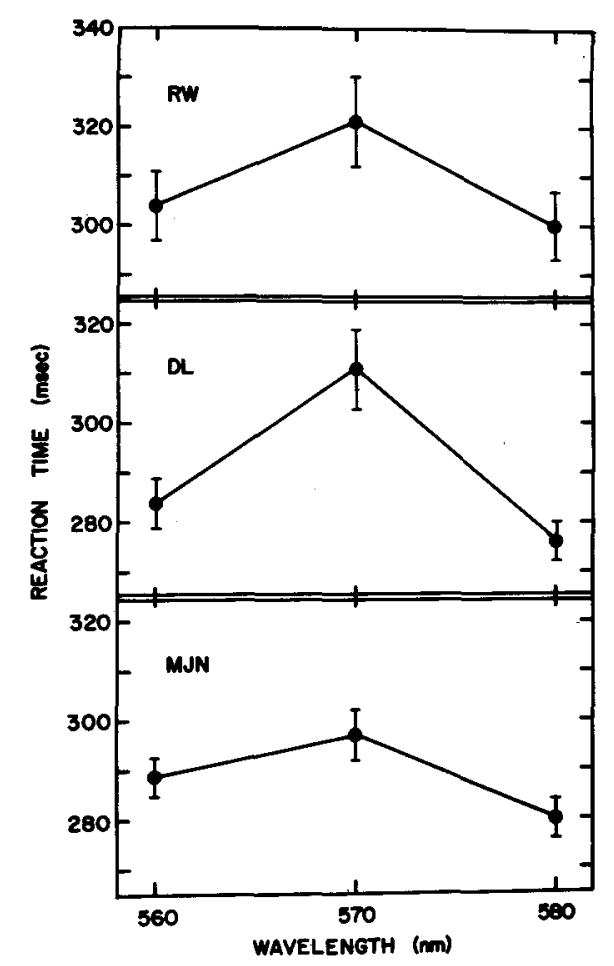

Figure 3. Individual mean reaction time as a function of wavelength in Experiment 2. Stimuli were presented in substitution.

\section{EXPERIMENT 3}

There is evidence that the receptor class whose maximal sensitivity is to blue light may have a longer integration time than those most sensitive to longer wavelengths (Brindley, DuCroz, \& Rushton, 1966; Krauskopf \& Mollon, 1971; Mollon \& Krauskopf, 1973; Regan \& Tyler, 1971). It is possible that these receptors would contribute minimally in a flicker procedure when chromatic stimuli are very brief, but that their contribution would increase disproportionately when stimulus duration is longer. The luminance matches for blue light produced by heterochromatic flicker photometry may not hold when these stimuli are presented for longer durations, as in the RT task. Specifically, the 430- and 460-nm stimuli used in Experiment 1 may have been at a higher luminance than required for substitution. Experiment 3 was designed to test that possibility.

The results of Experiment 1 suggested that RT to a chromatic stimulus is slowest when its luminance is the same as that of the background. If the flicker match for blue light is the appropriate luminance match for the RT task, then RT to blue light should be slowest at that luminance setting and faster when its luminance is decreased.

\section{Method}

Subjects. M.J.N. and K.H., both of whom had participated in Experiment 1, served as subjects.

Apparatus. The stimulus display and apparatus were the same as in Experiments 1 and 2. A single interference filter with peak transmission at $453 \mathrm{~nm}$ and a half-bandwidth transmission of $13 \mathrm{~nm}$ was used.

Procedure. The flicker match between the achromatic background and the 453-nm light was determined for each subject.

The procedure for the RT task was the same as in Experiments 1 and 2. RT was measured to $453-\mathrm{nm}$ stimuli at three luminance levels: the heterochromatic flicker photometric match and 0.1 and $0.2 \mathrm{log}$ unit below the match. The luminance was reduced by adding neutral density to the chromatic channel. The luminance of the background was constant.

After 1 day of practice, all three possible pairs of these three stimuli were used twice on each of 3 consecutive days. The order of blocks was controlled by a Latin square. There were 240 responses per stimulus per subject.

\section{Results}

Mean RT data for each subject are shown in Table 1. Both subjects responded most slowly to the luminance level determined by the flicker procedure. A three-way ANOVA (Luminance by Foreperiod by Days) indicated significant main effects of luminance $[F(2,2)=20.92, p<.05]$ and foreperiod $[\mathrm{F}(3,3)=25.21, \mathrm{p}<.01]$.

As the luminance of the 453-nm light is reduced below the flicker match setting, reaction time decreases. Pilot data indicated that, as expected, luminances higher than the flicker match also resulted in faster responses. These data confirm that 
Table 1

Mean Reaction Time to $453 \mathrm{~nm}$ at Three Luminance Levels

\begin{tabular}{cccc}
\hline Subject & $\begin{array}{c}\text { Flicker } \\
\text { Match }\end{array}$ & $\begin{array}{c}\text { Match } \\
-.1 \text { Log Unit }\end{array}$ & $\begin{array}{c}\text { Match } \\
-.2 \text { Log Unit }\end{array}$ \\
\hline M.J.N. & 239 & 225 & 221 \\
K.H. & 264 & 254 & 253 \\
\hline
\end{tabular}

the flicker match setting for blue light was the appropriate radiance to use in the substitution condition of the RT task. The fast responses to 430 and $460 \mathrm{~nm}$ in Experiment 1 did not result from the artifactual presence of a luminance increment.

\section{DISCUSSION}

These experiments show that when chromatic stimuli are presented on a background of lower luminance, there is no effect of wavelength on simple reaction time. Under those conditions subjects evidently base their responses on the luminance signal rather than the chromatic signal, a hypothesis supported by the fact that RT to a white light increment is the same as that to chromatic lights. When only a chromatic signal is present, as in substitution conditions, the effect of wavelength is substantial. A consideration of relevant physiological data may facilitate a better understanding of the effective difference between the two conditions and the form of the wavelength effect in the substitution condition.

Physiological theories of color vision postulate the existence of achromatic and chromatic processing channels in the visual system. The achromatic channel responds only to changes in luminance; the chromatic channel is sensitive to changes in both luminance and wavelength. Evidence for achromatic and chromatic channels has been found in the spectrally nonopponent and spectrally opponent cells, respectively, of the retina (Gouras, 1968) and lateral geniculate nucleus (DeValois, Abramov, \& Jacobs, 1966).

The presentation of a stimulus on a dimmer achromatic background should lead to activity in both processing channels. However, physiological data reported by Marrocco and Brown (1975) suggest that the achromatic channel will respond sooner. They found that nonopponent cells in the monkey lateral geniculate nucleus had significantly shorter latencies and faster conduction velocities than opponent cells. Assuming that responses in a speeded task are determined by the fastest processing channel, simple RT in an increment condition should be determined primarily by the achromatic channel. Further, because the size of the luminance signal, which is the effective stimulus for the achromatic channel, was matched for all wavelengths in Experiment 1, we would predict no wavelength effect in that condition.

The variation in $R T$ with wavelength in the sub- stitution condition is very similar to the variation in saturation with wavelength: the spectral region around $570 \mathrm{~nm}$ appears least saturated, and short wavelengths are somewhat more saturated than long wavelengths (Graham, 1965; Wright \& Pitt, 1937). In their work on saturation discrimination in the macaque monkey, DeValois and his colleagues (DeValois et al., 1966; DeValois \& Marrocco, 1973) determined the change in purity required to produce a criterion change in the firing rate of opponent cells in the lateral geniculate. The shape of this function closely paralleled that of the monkeys' behavioral saturation discrimination. DeValois and Marrocco (1973) propose that spectrally opponent cells provide an adequate mechanism for saturation discrimination. Also, Marrocco and DeValois (1977) recently showed that when chromatic stimuli are presented in a substitution condition, the neutral point of all types of opponent cells moves to near $570 \mathrm{~nm}$. A $570-\mathrm{nm}$ stimulus (which produced the slowest response times in the present study) elicits minimal response in opponent cells.

It seems that both the saturation of spectral lights and the speed with which they can be responsed to increase with the amount of activity elicited in chromatic processing channels. However, the dependence of RT on wavelength is concealed when stimulus conditions allow responses to be mediated by the faster achromatic channel.

\section{REFERENCE NOTE}

1. Bowen. R. W., Lindsey, D. T., \& Smith, V. C. Wavelength effects on temporal resolution. Paper presented at the meeting of ARVO. Sarasota, Florida, April 1977.

\section{REFERENCES}

Breton, M. E. Hue substitution: Wavelength latency effects. Vision Research, 1977, 17, 435-443.

Brundley, G. S., DuCroz, J. J., \& Rushton, W. A. H. The tlicker fusion frequency of the blue-sensitive mechanism of colour vision. Joumal of Physiology, 1966, 183, 497-500.

DeGroot. S. G., \& Gebhard, J. W. Pupil size as determined by adapting luminance. Journal of the Optical Society of America. 1952. 42. 492-495.

DFValots, R. L., Abramov, I., \& Jacobs, G. H. Analysis of response patterns of LGN cells. Journal of the Optical Society of America, 1966, 56, 966-977.

DeVAlois. R. L.. \& Marrocco. R. T. Single cell analysis of saturation discrimination in the macaque. Vision Research, 1973, 13. 701-711.

FinN. J. P.. \& LIT, A. Effect of photometrically matched wavelength on simple reaction time at scotopic and photopic levels of illumination. Proceedings. 79th Annual Convention. APA, 1971. 7. 5-6.

Gouras. P. Identification of cone mechanisms in monkey ganglion cells. Journal of Physiology, 1968, 199. 533-547.

Graham, C. H. Vision and visual perception. New York: Wiley, 1965.

Holmes. J. L. Reaction time to photometrically equal chromatic stimuli. American Journal of Psychology, 1926, 37. 414-417.

Jones. P. D. \& W WLKInson. H. Latency differences to mono- 
chromatic stimuli measured by disjunctive reaction time. Perceptual and Motor Skills, 1975, 41, 55-59.

KrausKopf, J., \& Mollon, J. D. The independence of the temporal integration properties of individual chromatic mechanisms in the human eye. Journal of Physiology, 1971, 219, 611-623.

Lit, A., Young, R. H., \& Shaffer, M. Simple reaction time as a function of luminance for various wavelengths. Perception \& Psychophysics, 1971, 10, 397-399.

Marrocco, R. T., \& Brown, J. B. Correlation of receptive field properties of monkey LGN cells with the conduction velocity of retinal afferent input. Brain Research, 1975, 92, 137-144.

MARRocco, R. T., \& DEVALoIs, R. L. Locus of spectral neutral point in monkey opponent cells depends on stimulus luminance relative to background. Brain Research, 1977, 119, 465-470.

Mollon, J. D., \& KRauskopf, J. Reaction time as a measure of the temporal response properties of individual colour mechanisms. Vision Research, 1973, 13, 27-40.
Pieron, H. La sensation chromatique. Données sur la latence propre et l'établissement des sensations de couleur. L'Année Psychologique, 1931, 32, 1-29.

Pollack, J. D. Reaction time to different wavelengths at various luminances. Perception \& Psychophysics, 1968, 3, 17-24.

RegAN, D., \& TYLER, C. W. Temporal summation and its limit for wavelength changes: An analogue of Bloch's law for color vision. Journal of the Optical Society of America, 1971, 61, 1414-1421.

WeINGarten, F. Wavelength effect on visual latency. Science, 1972, 176, 692-693.

WRIGHT, W. D., \& PitT, F. H. G. The saturation-discrimination of two trichromats. Proceedings of the Physical Society, 1937, 49, 329-331.

(Received for publication June 20, 1977; accepted September 7, 1977.) 